\title{
PERFIL EPIDEMIOLÓGICO DO CÂNCER DE PULMÃO E BRÔNQUIOS EM RELAÇÃO AO TIPO HISTOLÓGICO: UM ESTUDO EPIDEMIOLÓGICO NA CIDADE DE CASCAVEL-PR EM COMPARAÇÃO COM O PARANÁ
}

\section{EPIDEMIOLOGIC PROFILE OF LUNG AND BRONCHIAL CANCER IN RELATION TO THE HISTOLOGICAL TYPE: AN EPIDEMIOLOGICAL STUDY IN THE CITY OF CASCAVEL- PR COMPARED TO PARANA}

\author{
Luana Pantano Bergamin ${ }^{1}$, Kauana da Silveira ${ }^{1}$, Juliano Karvat de Oliveira ${ }^{2}$, Elaine \\ de Oliveira Zanini ${ }^{3}$
${ }^{1}$ Acadêmico do curso de Medicina do Centro Universitário FAG - Cascavel-PR. ${ }^{2}$ Mestre em Ciências Ambientais pela Universidade Estadual do Oeste do Paraná- UNIOESTE. Professor do Centro
Universitário FAG - Cascavel. ${ }^{3}$ Mestre em Desenvolvimento Regional e Agronegócio pela
Universidade Estadual do Oeste do Paraná - UNIOESTE. Professora do Centro Universitário FAG - Cascavel.
*Autor Correspondente: ezanini@fag.edu.br, https://orcid.org/0000-0002-5098-7857

DOI: $10.35984 /$ fjh.v2i1.166

\section{RESUMO}

O câncer representa um problema de saúde pública tanto nos países desenvolvidos como nos países em desenvolvimento, especialmente o câncer de pulmão. Apesar de sua incidência vir diminuindo desde a década de 1980 para os homens e desde os anos 2000 entre as mulheres, ele ainda é responsável por grande letalidade no Brasil. Devido a extrema importância desse câncer em relação a morbimortalidade no mundo, o presente artigo tem como finalidade apresentar um estudo epidemiológico que analisa os fatores de risco (escolaridade, sexo, histórico de consumo de tabaco, faixa etária, histórico familiar, raça/cor e tipo histológico), os quais contribuem para o câncer de pulmão e brônquios, na cidade de Cascavel e no estado do Paraná entre os anos 2013 a 2017.

Palavras-chave: Câncer de pulmão e brônquios. Fatores de risco. Perfil epidemiológico. Tipo histológico.

\begin{abstract}
Cancer represents a public health problem in both developed and developing countries, especially lung cancer. Although its incidence has been decreasing since the 1980s for men and since the 2000s among women, it is still responsible for high mortality in Brazil. Due to the extreme importance of this cancer in relation to morbidity and mortality worldwide, this article aims to present an epidemiological study that analyzes risk factors (education, gender, history of smoking, age group, family history, race / color and histological type), which contribute to lung and bronchial cancer in the city of Cascavel and in the state of Paraná between 2013 to 2017.
\end{abstract}

Keywords: Lung and bronchial cancer. Risk factors. Epidemiological profile. Histological type.

\section{INTRODUÇÃO}

O câncer, conhecido há vários séculos, é um crescimento celular incontrolável e anormal, o qual possui a capacidade de invadir os tecidos vizinhos e à distância (UEHARA, 1998, p. 266). O câncer de pulmão, um dos tumores malignos mais 
comuns, é o segundo mais incidente em homens e mulheres no Brasil, sendo que em relação ao mundo é o que mais contribui para as taxas de mortalidade desde 1985. Para comprovar tais dados, tem-se que, segundo o Instituto Nacional de Câncer, o número de mortes em 2017 foi de 27.931, sendo 16.139 homens e 11.792 mulheres (INCA, 2019).

Como principal causa de câncer de pulmão tem-se o tabagismo. Segundo Uehara (1998, p. 266), "Uma característica própria deste câncer de incidência alta de mortalidade é a sua causa, que é o hábito de fumar, o qual entretanto, possibilita uma ação profilática concreta e com bons resultados, que é o combate ao tabagismo".

Outros fatores causais são as infecções pulmonares, a exposição à poluição do ar, doenças pulmonares obstrutivas crônicas, fatores genéticos, idade avançada, exposição ocupacional a agentes químicos ou físicos (INCA, 2019).

Sobre a exposição a poluição do ar Zamboni (2002, p. 44) afirma que "[...] o indivíduo que vive nas grandes cidades tem risco maior de ser acometido pelo câncer do pulmão, possivelmente devido à maior exposição aos carcinógenos, tais como os hidrocarbonetos policíclicos e a fumaça do óleo diesel".

Observou-se também a influência da escolaridade sobre o consumo de tabaco, sendo confirmado existir maior número de tabagistas na população de menor escolaridade, principalmente entre homens mais jovens (GULNAR et al, 2009).

Ao tratar dos resultados da pesquisa, Gulnar (2009, p. 53) afirma que "Entre mulheres acima de 60 anos, prevalências maiores entre as de maior escolaridade podem expressar tendências de décadas passadas, nas quais fumar era símbolo de liberdade". Por outro lado, segundo Barros,

A maior parte dos casos acomete indivíduos entre 50 e 70 anos de idade e, embora fosse inicialmente uma doença epidêmica entre homens em nações industrializadas, o câncer de pulmão tornou-se uma doença cada vez mais comum entre as mulheres (BARROS et al, 2006, p. 222).

De acordo com Zamboni (2002, p.41), o câncer de pulmão, no início do século $\mathrm{XX}$ era considerado doença rara, tornando-se no final desse mesmo século a neoplasia mais letal em todo o mundo e uma das principais causas evitáveis de morte, tendo o tabagismo contribuição importante para o aumento nas estatísticas de mortalidade (INCA, 2019).

[...] O fumo pode aumentar o risco de morte de 20 a 30 vezes em tabagistas de longa data e de 30 a $50 \%$ em fumantes passivos. As taxas de incidência de câncer de pulmão em um determinado país refletem o consumo de cigarros. Na população masculina, o hábito de fumar continua responsável pela maioria dos casos diagnosticados de câncer de pulmão, que permanece como uma doença letal. (MALTA, 2007, p. 537).

Na pesquisa realizada por Zamboni no ano de 2002 foi investigada a influência do tabaco sobre os fumantes passivos, analisando o risco de aparecimento de câncer do pulmão entre casados, quando apenas um dos cônjuges fumava. Hackshaw et al (1997), demonstram, dessa forma, que há grande probabilidade de fumantes passivos que convivem com fumantes ativos desenvolverem câncer de pulmão e brônquios. Segundo ele, "Esse risco cresce com o maior número de cigarros fumados pelo cônjuge e com a duração da exposição".

Ainda, é notório que há efeito dos fatores socioeconômicos sobre os desfechos na saúde, devido a fatores comportamentais e clínicos. Desse modo, Gulnar afirma que,

A prevalência de fumantes foi maior entre indivíduos de baixa escolaridade.

$\mathrm{E}$ de fato, a prevalência de tabagismo no Brasil está diminuindo, FAG Journal of Health - ISSN 2674-550X, 2020, v.2, n.1, p. 143 
especialmente na população mais jovem de melhor condição socioeconômica. Isso sugere que a política de controle do tabagismo no Brasil tem sido efetiva para prevenir a iniciação de jovens, porém deve ter atingido mais diretamente os extratos de melhor nível socioeconômico. (GULNAR et al, 2009, p. 52).

Deve-se acrescentar também que "há [...] diferenças relevantes entre a prevalência de homens e mulheres. Estima-se que a prevalência entre os homens seja de cerca de dez vezes maior do que entre as mulheres (PAES, 2015, p. 54)". Uma provável explicação para esse fato se relaciona aos diferentes hábitos tabágicos observados entre os sexos, já que, segundo estudos, as mulheres tendem a começar a fumar com idade mais avançada, fumam menos cigarros diariamente e não tragam profundamente.

Em relação a influência do histórico familiar, Barros (2006, p. 223) obteve em sua pesquisa realizada com 263 pacientes, que a "História familiar de câncer foi positiva em 73 casos (28\%), sendo que em 16 deles a neoplasia pulmonar foi relatada (6\%)".

Quanto ao tipo histológico, os quatro diferentes tipos de câncer do pulmão são o adenocarcinoma, o carcinoma de pequenas células, o escamoso e o carcinoma de grandes células. Com relação a isso é afirmado que,

Atualmente, [...] tanto o carcinoma escamoso quanto o carcinoma indiferenciado de pequenas células e o adenocarcinoma estão relacionados com o tabagismo. A maioria dos carcinógenos ocupacionais dá origem a tumores do pulmão com distribuição histológica semelhante àquela causada pela fumaça do tabaco.s.'spe: (ZAMBONI, 2002, p.41).

Sobre o adenocarcinoma, "Em praticamente todas as avaliações realizadas desde a década de 80, a incidência desse último tipo de câncer do pulmão foi maior no sexo feminino e vem aumentando progressivamente" (ZAMBONI, 2002, p. 42).
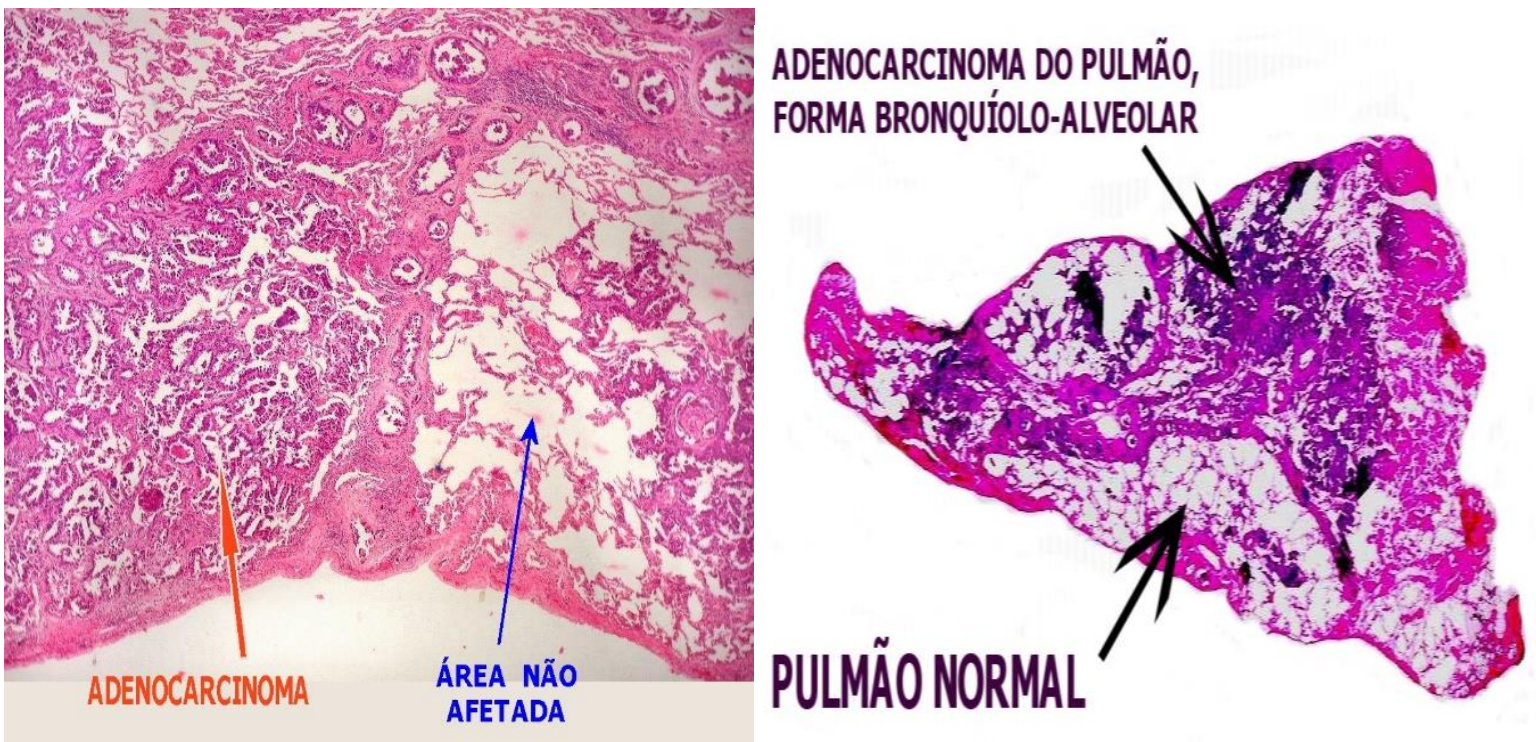

Figura 1. Adenocarcinoma de Pulmão (tipo histológico). FONTE: ATLAS UNICAMP (2016)

Em relação a raça/cor os estudos apontam que,

[...] no Brasil, os negros possuem menor escolaridade, menor salário, residem nos bairros de periferia das grandes cidades e estão excluídos de vários direitos sociais. Parte-se da hipótese de que o processo saúde, doença e morte é construído socialmente e demarcado pelo espaço social que homens 
e mulheres, brancos e negros, ocupam na sociedade. (BATISTA, 2004).

Sendo assim, vale ressaltar que há fatores que condicionam e interferem na adoção de estilos de vida saudáveis e que as diversas medidas de controle do tabagismo alcançam homens e mulheres de diferentes níveis de escolaridade e esferas sociais.

\section{METODOLOGIA}

Esta pesquisa caracteriza-se como pesquisa bibliográfica baseando-se em artigos, revistas, sites. Segundo Gil, essa pesquisa,

[...] é desenvolvida com base em material já elaborado, constituído principalmente de livros e artigos científicos. Embora em quase todos os estudos seja exigido algum tipo de trabalho dessa natureza, há pesquisas desenvolvidas exclusivamente a partir de fontes bibliográficas. Boa parte dos estudos exploratórios pode ser definida como pesquisas bibliográficas. As pesquisas sobre ideologias, bem como aquelas que se propõem a uma análise das diversas posições acerca de um problema, também costumam ser desenvolvida quase exclusivamente mediante fontes bibliográficas. (GIL, 2019, p.3).

Foi utilizado como fonte os dados disponíveis na plataforma do INCA (Instituto Nacional do Câncer), para realizar um estudo retrospectivo de 2013 a 2017, comparando-se o tipo histológico do câncer de pulmão e brônquios com categorias demográficas e comportamentais: escolaridade, sexo, histórico de consumo de tabaco, faixa etária, histórico familiar, raça/cor e tipo histológico, com o objetivo de delinear o perfil epidemiológico dos portadores desse câncer na cidade de Cascavel - PR, em comparação com o estado do Paraná.

Em relação aos resultados essa pesquisa classifica-se como quantitativa e qualitativa. Quantitativa por apresentar percentuais e qualitativa por analisar vários fatores de risco para o desenvolvimento do câncer de pulmão e brônquios.

Com base nos ensinamentos de Richardson, Dalfovo menciona que o método quantitativo,

[...] caracteriza-se pelo emprego da quantificação, tanto nas modalidades de coleta de informações, quanto no tratamento dessas através de técnicas estatísticas, desde as mais simples até as mais complexas. Conforme supra mencionado, ele possui como diferencial a intenção de garantir a precisão dos trabalhos realizados, conduzindo a um resultando com poucas chances de distorções (DALFOVO, 2008, p.7).

Por outro lado, a pesquisa qualitativa, de acordo com Dalfovo,"[...] é aquela que trabalha predominantemente com dados qualitativos, isto é, a informação coletada pelo pesquisador não é expressa em números, ou então os números e as conclusões neles baseadas representam um papel menor na análise" (DALFOVO, 2008, p.9).

\section{RESULTADOS E DISCUSSÃO}

Fatores analisados: Histórico de consumo de tabaco (consumidores e exconsumidores); Histórico familiar (presente); Escolaridade (ensino fundamental incompleto); Tipo histológico (adenocarcinoma); Faixa etária (acima de 55 anos); Sexo (masculino); Raça/cor (branca). Na figura 2 estão descritos apresentados os fatores de risco para câncer de pulmão e brônquios. 


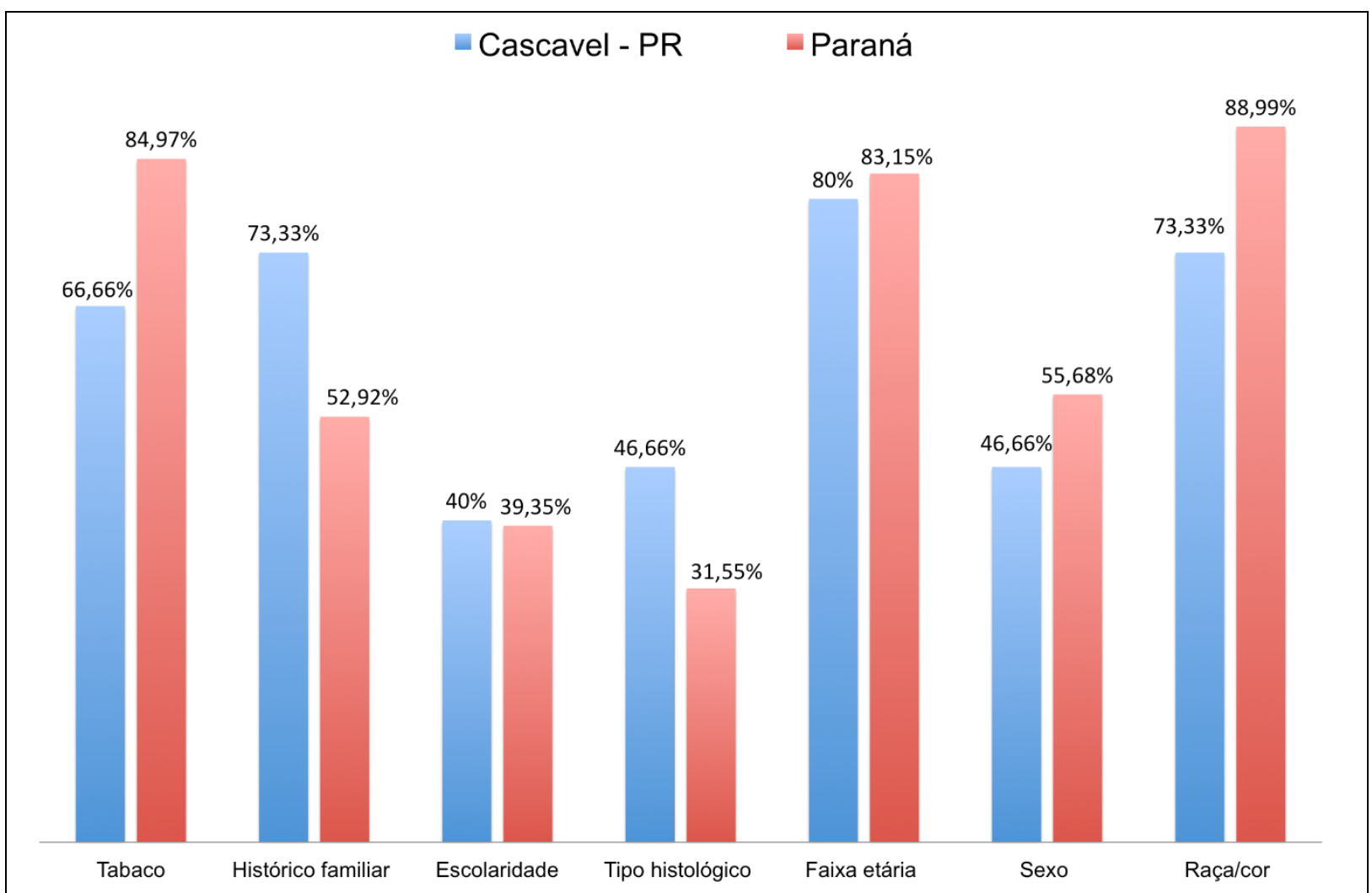

Figura 2. Fatores de risco para o Câncer de Pulmão e Brônquios. FONTE: Elaborado pelos autores, adaptado do INCA (2019).

Diante do exposto, tem-se o tabagismo como principal causa de câncer de pulmão. Os resultados obtidos em relação ao consumo de tabaco evidenciam que em grande parte dos casos diagnosticados, o câncer de pulmão está associado ao consumo de derivados de tabaco.

Outros fatores causais predominantes são o sexo, em que no caso de Cascavel, os dados contrariaram o conhecimento já estabelecido de que homens tem mais tendência a desenvolver o câncer de pulmão. Por outro lado, no Paraná tal fato foi comprovado.

Os dados obtidos em relação ao sexo, confirmam que a diferença se deve aos padrões de adesão e cessação do tabagismo constatado em ambos os sexos. Entretanto, a tentativa de parar de fumar é maior no sexo feminino, demonstrando que homens e mulheres apresentam condutas de saúde diferentes.

Além disso, os dados encontrados ratificam que indivíduos de menor escolaridade apresentaram maior prevalência de tabagismo, concluindo ser a escolaridade um fator protetor. Ainda, observou-se existir maior número de tabagistas na população de menor escolaridade.

Em relação a faixa etária, teve prevalência em idade mais avançada. Demonstrou-se também considerável influência do histórico familiar e o tipo histológico predominante foi o adenocarcinoma, seguido do carcinoma escamocelular. Em relação a raça, é provável que a cor branca foi predominante na pesquisa realizada, devido a sua prevalência na região Sul, inclusive no Paraná. 


\section{CONCLUSÃO}

Considerando-se o sexo, observou-se que a tributação é um elemento que faz parte da decisão de homens e mulheres na compra do cigarro, embora se perceba serem as mulheres mais sensíveis a preços, fatores culturais e sociais, incluindo a maior preocupação do sexo feminino com os cuidados em saúde e higiene pessoal, relacionado ao odor desagradável que a nicotina causa, por exemplo. Além disso, os resultados ressaltam a relevância do aperfeiçoamento de estratégias direcionadas às mulheres e a continuidade das ações de manejo do tabagismo para homens.

Conclui-se, em relação aos dados apresentados, que algumas práticas e hábitos de vida podem contribuir para a prevenção, como evitar o tabagismo ativo e passivo, bem como promover projetos educacionais eficientes no combate ao consumo de tabaco. É comprovada a importância de tal medida devido ao fato de que cerca de $80 \%$ dos fumantes começam a fumar antes dos 18 anos, sendo o tabagismo considerado uma doença pediátrica.

\section{REFERÊNCIAS}

BARROS, J. A. et al. Diagnóstico precoce do câncer de pulmão: o grande desafio. Variáveis epidemiológicas e clínicas, estadiamento e tratamento. J. Bras Pneumol, p. 221-227, 2006.

BATISTA, L.E. Masculinidade, raça/cor e saúde, Setembro 2004.

DALFOVO, Michael Samir; LANA, Rogério Adilson; SILVEIRA, Amélia. Métodos quantitativos e qualitativos: um resgate teórico. Revista Interdisciplinar Científica Aplicada, Blumenau, v.2, n.4, p.01-13, Sem II. 2008

GIL. A.C. Como classificar as pesquisas?. Disponível em http://www.madani.adv.br/aula/Frederico/GIL.pdf. Acesso em: 14 nov. 2019.

INCA - Instituto Nacional de Câncer. Tipos de câncer: Câncer de pulmão. Disponível em https://www.inca.gov.br/tipos-de-cancer/cancer-de-pulmao. Acesso em: 28 set. 2019.

HACKSHAW AK, LAW MR, WALD NJ. The accumulated evidence on lung cancer and environmental tobacco smoke. BMJ 1997.

MALTA, D. C. et al. Tendência de mortalidade do câncer de pulmão, traquéia e brônquios no Brasil, 1980-2003. J. Bras Pneumol, p. 536-543, Janeiro 2007.

OLIVEIRA, C. M. D.; GORAYEB, R. Diferenças de gênero e fatores motivacionais para início do tabagismo em adolescentes. Rev. Saúde \& Transformação Social, v. 3, p. 49-54, 2012.

PAES, N. L. Fatores econômicos e diferenças de gênero na prevalência do tabagismo em adultos. Rev. Ciência \& Saúde Coletiva, p. 53-61, 2015. https://doi.org/10.1590/1413-81232015211.00162015

RICHARDSON, Roberto Jarry. Pesquisa social: métodos e técnicas. São Paulo: Atlas, 1989. 
SILVA, G. A. E. Diferenças de gênero na tendência de mortalidade por câncer de pulmão nas macrorregiões brasileiras. Rev. Brasileira Epidemiol, p. 411-419, Maio 2008.

SILVA, G. A. E. Tabagismo e escolaridade no Brasil, 2006. Rev. Saúde Pública, p. 48-56, Agosto 2009.

UEHARA C; JAMNIK S \& SANTORO IL. Câncer de pulmão. Medicina, Ribeirão Preto, 31: 266-276, abr./jun. 1998

UNICAMP - Anatomia Patológica. Adenocarcinoma de pulmão. Disponível em http://anatpat.unicamp.br/lamneo13.html. Acesso em: 14 nov. 2019.

ZAMBONI, M. Epidemiologia do câncer do pulmão. J. Pneumol, p. 41-47, jan-fev 2002. 